\title{
Obesity dependent metabolic signatures associated with nonalcoholic fatty liver disease progression
}

\author{
J. Barr ${ }^{1}$, J. Caballería ${ }^{2}$, I. Martínez-Arranz ${ }^{1}$, A. Domínguez-Díez ${ }^{3}$, C. Alonso ${ }^{1}$, J. Muntané ${ }^{4}$, \\ M. Pérez-Cormenzana ${ }^{1}$, C. García-Monzón ${ }^{5}$, R. Mayo ${ }^{1}$, A. Martín-Duce ${ }^{6}$, M. Romero- \\ Gómez $^{7}$, O. Lo lacono ${ }^{8}$, J. Tordjman ${ }^{9}$, R.J. Andrade ${ }^{10}$, M. Pérez-Carreras ${ }^{11}$, Y. Le \\ Marchand-Brustel ${ }^{12}$, A. Tran ${ }^{12}$, C. Fernández-Escalante ${ }^{3}$, E. Arévalo ${ }^{4}$, M. García-Unzueta $^{3}$, \\ K. Clement ${ }^{9}$, J. Crespo ${ }^{3}$, P. Gual ${ }^{12}$, M. Gómez-Fleitas ${ }^{3}$, M.L. Martínez-Chantar ${ }^{13}$, A. Castro ${ }^{1}$, \\ S.C. $\mathbf{L u}^{14}, \mathbf{M}$. Vázquez-Chantada ${ }^{1}$, and J.M. Mato ${ }^{13}{ }^{*}$ \\ ${ }^{1}$ OWL Genomics, Derio, Bizkaia, Spain \\ ${ }^{2}$ Liver Unit, Hospital Clínic, Centro de Investigación Biomédica en Red de Enfermedades \\ Hepáticas y Digestivas (CIBERehd); Institut d'Investigacions Biomediques August Pi Sunyer \\ (IDIBAPS), Barcelona, Spain \\ ${ }^{3}$ Servicio de Cirugía General, Servicio de Aparato Digestivo, Servicio de Análisis Clínicos, \\ Hospital Universitario Marqués de Valdecilla, Universidad de Cantabria, Santander, Spain \\ ${ }^{4}$ Liver Research Unit, Surgery Department IMIBIC (Instituto Maimónides para la Investigación \\ Biomédica de Córdoba), "Reina Sofía" University Hospital, Córdoba, Spain; CIBERehd; Instituto \\ de Salud Carlos III, Spain \\ ${ }^{5}$ Liver Research Unit, Instituto de Investigación Sanitaria Princesa, University Hospital Santa \\ Cristina; CIBERehd, Madrid, Spain \\ ${ }^{6}$ Departamento de Enfermería, Hospital Universitario Príncipe de Asturias; Alcalá de Henares \\ University, Madrid, Spain \\ ${ }^{7}$ UG MQ Enf. Digestivas, Hospital Universitario Nuestra Señora de Valme, Universidad de \\ Sevilla; CIBERehd, Sevilla, Spain \\ ${ }^{8}$ Sección de Aparato Digestivo, Hospital del Tajo, Aranjuez, Spain \\ 9 ICAN Institut Cardiométabolisme et Nutrition, Paris, Pitié-Salpêtrière Hospital, Paris, France; \\ INSERM, U872, Nutriomique, Paris, France; Université Pierre et Marie Curie-Paris, Centre de \\ Recherche des Cordeliers, UMR S 872, Paris, France \\ ${ }^{10}$ Hepatology Section, Hospital Clínico Virgen de la Victoria; CIBERehd, Málaga, Spain \\ ${ }^{11}$ Medicina Aparato Digestivo, Hospital Universitario “12 de Octubre”, Madrid, Spain \\ ${ }^{12}$ INSERM, U895, Team 8 «Hepatic complications in obesity»; University of Nice-Sophia- \\ Antipolis, Faculty of Medicine; Centre Hospitalier Universitaire of Nice, Digestive Center, Nice, \\ France \\ ${ }^{13} \mathrm{CIC}$ bioGUNE, CIBERehd, Derio, Bizkaia, Spain
}

\footnotetext{
"Corresponding author: José M. Mato, CIC bioGUNE, CIBERehd, Bizkaia Technology Park, 48160 Derio, Bizkaia, Spain. director@ cicbiogune.es; Tel: +34-944-061300; Fax: +34-944-061301.

Associated Content

Supporting Information

Supplementary tables and figures. This material is available free of charge via the Internet at http://pubs.acs.org.
} 
${ }^{14}$ Division of Gastrointestinal and Liver Diseases, USC Research Center for Liver Diseases, Southern California Research Center for Alcoholic Liver and Pancreatic Diseases and Cirrhosis, Keck School of Medicine, University of Southern California, Los Angeles, California 90033

\section{Abstract}

Our understanding of the mechanisms by which nonalcoholic fatty liver disease (NAFLD) progresses from simple steatosis to steatohepatitis (NASH) is still very limited. Despite the growing number of studies linking the disease with altered serum metabolite levels, an obstacle to the development of metabolome-based NAFLD predictors has been the lack of large cohort data from biopsy-proven patients matched for key metabolic features such as obesity. We studied 467 biopsied individuals with normal liver histology $(\mathrm{n}=90)$ or diagnosed with NAFLD (steatosis, $\mathrm{n}=246$; NASH, $\mathrm{n}=131$ ), randomly divided into estimation ( $80 \%$ of all patients) and validation (20\% of all patients) groups. Qualitative determinations of 540 serum metabolite variables were performed using ultra-performance liquid chromatography coupled to mass spectrometry (UPLCMS). The metabolic profile was dependent on patient body-mass index (BMI), suggesting that the NAFLD pathogenesis mechanism may be quite different depending on an individual's level of obesity. A BMI-stratified multivariate model based on the NAFLD serum metabolic profile was used to separate patients with and without NASH. The area under the receiver operating characteristic curve was 0.87 in the estimation and 0.85 in the validation group. The cutoff $(0.54)$ corresponding to maximum average diagnostic accuracy (0.82) predicted NASH with a sensitivity of 0.71 and a specificity of 0.92 (negative/positive predictive values $=0.82 / 0.84$ ).

The present data, indicating that a BMI-dependent serum metabolic profile may be able to reliably distinguish NASH from steatosis patients, have significant implications for the development of NASH biomarkers and potential novel targets for therapeutic intervention.

\section{Keywords}

NAFLD; steatosis; NASH; metabolomics; biomarkers

\section{INTRODUCTION}

Most body fat is stored as triacylglycerides (TAG) in white adipose tissue. Impaired lipid buffering within the enlarged adipocyte leads to the exposure of nonadipose tissues such as the liver to an increased influx of nonesterified fatty acids (NEFA) ${ }^{1}$. Such metabolic stress can lead to an imbalance between NEFA supply and hepatic NEFA disposal pathways, causing excess lipids to accumulate in the form of TAG, a condition known as nonalcoholic fatty liver disease (NAFLD) ${ }^{2,3}$. Further lipid excess in the sensitized fatty liver is metabolized through alternative pathways that result in the production of toxic reactive species, increased oxidative stress, and disturbances in membrane phospholipid composition - all of which are processes that may contribute to the progression of NAFLD through nonalcoholic steatohepatitis (NASH) ${ }^{2,4,5}$, which can then lead to cirrhosis and hepatocellular carcinoma ${ }^{2,6,7}$.

While obesity is well established as the main cause of NAFLD, we do not know why fatty liver also develops in lean subjects ${ }^{8,9}$. Our understanding of why only a relatively small proportion of NAFLD patients develop NASH is also very limited ${ }^{2,4,5}$. Modern widecoverage metabolite profiling technologies provide access to portions of biomolecular space that sample genetic, environmental and lifestyle factors which may help to explain some of these observations ${ }^{10,11}$. The technique is particularly well suited to liver injury assessment applications, where serum or urine are the most common types of sample made available for 
laboratory tests, as opposed to other disease scenarios, such as cancer, where tissue is readily made available for transcriptomic and proteomic analysis.

A recent study by Kalhan et al. ${ }^{12}$ compared the plasma metabolome in nonbiopsied lean controls with that found in obese NAFLD patients, showing no discernible differentiation between steatosis and NASH plasma. Puri et al. analyzed a similar set of patients, focusing on the plasma lipidome by quantifying derivatized fatty acids after lipid hydrolysis ${ }^{13}$ to reveal new evidence for the role of fatty acid traffic directed away from TAG synthesis in disease pathogenesis ${ }^{12,13}$. Our group has set up multiple ultra-performance liquid chromatography coupled to mass spectrometry (UPLC-MS) based platforms able to qualitatively analyze fatty acid derived moieties in their intact form as serum lipids, covering a wide range of molecular species also including bile acids, nonesterified fatty acids, and amino acids. The techniques are particularly useful for profiling subtle molecular diversity, over species such as for example ether-/acyl- linked glycerophospholipids, or the various subclasses of sphingolipids. These specific elements of the lipidome are potentially important role-players in the context of modern lipotoxic models of metabolic syndrome related disease pathogenesis, which may not be captured efficiently by the lipid hydrolysis/ fatty acid analysis methodology. This approach was used recently to describe common serum metabolic alterations observed in a NAFLD knock-out animal model and a smallscale cohort of morbidly obese human NAFLD patients, closely matched in key clinical features such as gender, age, and body-mass index (BMI ${ }^{14}$. Encouraged by these results we have implemented recently published ${ }^{15}$ analytical processing methods that allow qualitative LC-MS metabolite profiling to be performed over multiple analysis batches, thus permitting the inclusion of large cohorts of human patient samples suitable for clinical studies. The aim of the current study was to characterize the serum NAFLD metabolic profile as a function of patient BMI, exploring the differences among histological groups of patients with different grades of obesity.

\section{MATERIALS AND METHODS}

\subsection{Chemicals}

HPLC/MS-grade solvents were purchased from Sigma Aldrich (St. Louis, MO). Reference metabolite standard compounds were obtained from Sigma Aldrich, Avanti Polar Lipids (Alabaster, AL), and Larodan Fine Chemicals (Malmö, Sweden).

\subsection{Patients}

A total of 467 biopsied patients seen at 11 participating hospitals were recruited for the study using the following inclusion criteria: (1) age 18-75 years; (2) no known acute or chronic disease except for obesity or type 2 diabetes based on medical history, physical examination, and standard laboratory tests; (3) alcohol consumption was less than $20 \mathrm{~g} / \mathrm{day}$ for women and $30 \mathrm{~g} /$ day for men. Exclusion criteria included viral- and drug-induced causes of liver disease. All of the subjects were of Caucasian origin. The institutional review board at each of the participating hospitals approved the study and written informed consent was obtained from all patients. For all subjects diagnoses were established histologically in liver biopsy specimens. The reason for liver biopsy was either incidental (during laparoscopic cholecystectomy, $n=69$; or bariatric surgery, $n=311$ ) or suspected NAFLD, $n=87$. Currently there are no established guidelines on when to perform a liver biopsy in either adult or pediatric patients with suspected NAFLD. In the current population the decision was made on an individual basis by the treating gastroenterologist, independent of the present study. In most cases, the decision for biopsy indication was based on the presence of persistently abnormal liver enzymes or radiological evidence of a fatty liver along with negative testing for other common etiologies of liver disease. The histological diagnosis of NAFLD was 
established according to the criteria of a single liver pathologist in each participating hospital, guided by the scoring system defined by Kleiner et al. ${ }^{16}$. Following assessment, patients were classified by the pathologists into three histological groups: (1) Normal liver, (2) Steatosis (hepatic steatosis alone), and (3) NASH (presence as determined by the pathologist). For all subjects blood was drawn on the morning a liver biopsy was performed, following a minimum 8 hour, overnight fast. Serum was separated and stored at $-80{ }^{\circ} \mathrm{C}$ until analysis. Clinical data (Table 1) were collected retrospectively using patient records and laboratory values obtained at the time of surgery.

\subsection{Sample preparation and UPLC-MS analysis}

A UPLC-single quadrupole-MS amino acid analysis system was combined with two separate UPLC-time-of-flight (TOF)-MS based platforms analyzing methanol and chloroform/methanol serum extracts. Identified ion features in the methanol extract platform included NEFA, acyl carnitines, bile acids, monoacylglycerophospholipids, monoetherglycerophospholipids, free sphingoid bases, and oxidized fatty acids. The chloroform/methanol extract platform provided coverage over glycerolipids, cholesterol esters, sphingolipids, diacylglycerophospholipids, and acyl-ether-glycerophospholipids. The metabolite extraction procedure was as follows for each platform (lipid nomenclature follows the LIPID MAPS convention - www.lipidmaps.org):

Platform 1 - Methanol extract. Proteins were precipitated from the defrosted serum samples ( $75 \mu \mathrm{L}$ ) by adding 4 volumes of methanol in $1.5 \mathrm{~mL}$ microtubes at room temperature. The methanol used for extraction was spiked with the following compounds, chosen to encompass most of the retention time- $\mathrm{m} / \mathrm{z}$ space covered by the method, while being undetected in nonspiked human serum extracts: tryptophan$\mathrm{d}_{5}$ (indole- $\left.\mathrm{d}_{5}\right), \mathrm{PC}(13: 0 / 0: 0), \mathrm{NEFA}(19: 0)$, and dehydrocholic acid. After brief vortex mixing the samples were incubated overnight at $-20^{\circ} \mathrm{C}$. Supernatants $(300 \mu \mathrm{L})$ were collected after centrifugation at $16,000 \mathrm{xg}$ for 15 minutes, dried and reconstituted in 75 $\mu \mathrm{L}$ methanol, before being transferred to vials for UPLC-MS analysis on an AcquityLCTXE premier system (Waters Corp., Milford, MA).

Platform 2 - Amino acids. $10 \mu \mathrm{L}$ aliquots of the extracts prepared for platform 1 were derivatized for amino acid analysis ${ }^{17}$ on an Acquity-SQD system (Waters Corp.).

Platform 3 - Chloroform/Methanol extract: $10 \mu \mathrm{L}$ serum extracts were mixed with 10 $\mu \mathrm{L}$ sodium chloride $(50 \mathrm{mM})$ and $110 \mu \mathrm{L}$ of chloroform/methanol $(2: 1)$ in $1.5 \mathrm{~mL}$ microtubes at room temperature. The extraction solvent was spiked with the following compounds not detected in nonspiked human serum extracts: $\operatorname{SM}(\mathrm{d} 18: 1 / 6: 0)$, PE(17:0/17:0), PC(19:0/19:0), DAG(14:0/12:0), TAG(13:0/13:0/13:0), TAG(17:0/17:0/17:0), Cer(d18:1/17:0), ChoE(12:0). After brief vortex mixing the samples were incubated for 1 hour at $-20{ }^{\circ} \mathrm{C}$. After centrifugation at $16,000 \mathrm{x}$ g for 15 minutes, $70 \mu \mathrm{L}$ of the lower organic phase was collected and the solvent removed. The dried extracts were then reconstituted in $100 \mu \mathrm{L}$ acetronitrile/isopropanol (50:50), centrifuged (16,000 x $\mathrm{g}$ for 5 minutes), and transferred to vials for UPLC-MS analysis on an Acquity-SYNAPT G2 system (Waters Corp.).

Chromatographic separation and mass spectrometric detection conditions employed for each platform are summarized in Supplementary Table 1. Representative base peak ion chromatograms corresponding to the UPLC-TOF platforms are shown in Figure 1. Online tandem mass spectrometry (MS/MS) experiments for metabolite identification were performed on a Waters QTOF Premier (Waters Corp.) and a Waters SYNAPT G2 instrument, operating in both the positive and negative ion electrospray modes, as described in detail previously ${ }^{14}$. 


\subsection{Metabolite identification}

LCMS features (as defined by retention time, mass-to-charge ratio pairs, $R t-m / z$ ), were associated with NEFA, bile acids, oxidized fatty acids, free sphingoid bases, and amino acids by comparison of their accurate mass spectra and chromatographic retention times in commercial serum metabolite extracts (Promocell inc., Germany) with those obtained using available reference standards. A metabolite with $\mathrm{m} / \mathrm{z}$ value between 400 and $1000 \mathrm{Da}$ was considered unambiguously identified when $R t$ difference with respect to the standard was smaller than $3 \mathrm{~s}$ and the deviation from its $\mathrm{m} / \mathrm{z}$ value $(\delta \mathrm{m} / \mathrm{z})$ smaller than $3 \mathrm{ppm}$. For metabolites with $\mathrm{m} / \mathrm{z}$ values smaller than $400 \mathrm{Da}$, the criterion followed with respect to $R t$ was the same, but $\delta \mathrm{m} / z$ limit was set to $1.2 \mathrm{mDa}$. For all other species [acyl carnitines (AC), diacylglycerides (DAG), TAG, cholesterol esters (ChoE), glycerophosphocholine (PC), glycerophosphoethanolamine (PE), glycerophosphoinositol (PI), sphingomyelin (SM), ceramides (Cer), and monohexosylceramides $(\mathrm{CMH})]$ a theoretical $\mathrm{m} / \mathrm{z}$ database was first generated for all possible combinations of fatty acid derived moieties. The association of detected $R t-m / z$ pairs with lipid species contained in the theoretical database was subsequently established either by comparison of their accurate mass spectra and chromatographic retention times with those obtained using available reference standards or, where these were not available, by accurate mass MS/MS fragment ion analysis, as described in detail previously ${ }^{14,18}$ (mass fragment accuracy was $<3$ ppm for m/z 400-1000, and $<1.2 \mathrm{mDa}$ for $\mathrm{m} / \mathrm{z} 50-400$ ). A complete spectral library, containing relevant MS/MS data for all metabolites reported in this work is available on request from the authors.

The nontargeted LC/MS peak-detection software platform MarkerLynx (Waters Corp.) was used to analyse $R t-m / z$ pairs from the UPLC-TOF platforms that could not be confidently identified. Resulting peak lists were first deadducted and filtered according to peak area coefficients of variation $(\% \mathrm{CV}<20)$ recorded over 10 repeat standard serum extracts (Promocell inc.). For metabolic classes with members identified in more than one platform, the appropriate platform was assigned based on their physicochemical properties. For example, even when monoacyl- and monoether-glycerophospholipids were detected in both Platforms 1 and 3, the methanol extraction used in Platform 1 provided a more extensive coverage of the classes. Only the information provided by the selected platform was used for the downstream analysis, avoiding duplicities in the downstream analysis. The final list of unidentified variables was further reduced for each platform such that only variables with intensities at least twice those of any other peak detected in the same extracted ion chromatogram $(\mathrm{m} / \mathrm{z}$ window $\pm 0.02 \mathrm{Da})$ were included for analysis. Where possible, tentative metabolite identifications were assigned to unidentified variables using online (www.hmdb.ca) accurate mass database searching against their spectrally derived neutral molecular mass values.

A total of 540 variables were submitted for data processing (Identified/Unidentified: Platform $1=157 / 68$, Platform $2=20$, Platform $3=256 / 39$ ). Analytical and chemical structural details are provided for each variable in Supplementary Table 2A.

\subsection{Data processing and normalization}

All data were processed using the TargetLynx application manager for MassLynx 4.1 (Waters Corp.). The complete set of predefined $R t-\mathrm{m} / z$ pairs was fed into the software, which generated associated extracted ion chromatograms (mass tolerance window $=0.05$ Da), peak-detected and noise-reduced in both the LC and MS domains. A list of chromatographic peak areas was then generated for each sample injection, using the $R t-m / z$ pairs (retention time tolerance $=6 \mathrm{~s}$ ) as identifiers. 
Intra- and inter-batch normalization followed the procedure first described by van der Kloet et al. ${ }^{15}$. This process involved (i) multiple internal standard response correction (intra-batch normalization) and (ii) variable specific inter-batch single point external calibration using repeat extracts of a commercial serum sample (inter-batch normalization).

Each of the 6 analysis batches contained a maximum of 80 study samples that were accompanied by 10 repeat extracts of a commercial serum sample (Promocell inc.) used for single point inter-batch calibration (QC Calibration Sample ${ }^{15}$ ) and 5 repeat extracts of a second commercially obtained serum sample (Sigma Aldrich, St. Louis, MO) used to monitor the reproducibility of the overall analysis process (QC Validation Sample ${ }^{15}$ ). For each of the three analytical platforms, randomized duplicate sample injections (maximum number of study sample injections per batch $=160$ ) were performed, with each of the QC calibration and validation extracts uniformly interspersed throughout the entire batch run (maximum total number of injections per batch $=176$ ). Each batch run was preceded by a series of repeat full-loop of injections of a QC calibration extract, used to equilibrate the UPLC-MS systems $(\times 10$, or $\times 50$ if the batch followed system maintenance).

Van der Kloet et al. ${ }^{15}$ defined the optimal internal standard (IS) for each variable, $p$, as that which resulted in a minimum relative standard deviation (RSD) of the corrected response, $X$ ', calculated using all QC calibration samples over multiple batches (1).

$$
R S D_{p, q c}=\frac{\sigma_{X_{p, q c}^{\prime}}}{<X_{p, q c}^{\prime}>}
$$

However, for most single point, chemically similar internal standard correction MS-based methods, the average corrected response, $\left\langle X_{p, q c}^{\prime}\right.$, would be expected to differ from batch to batch, even if identical concentrations of internal standards were to be used in each batch. A number of experimental factors could cause these, generally small, differences - such as for example changes in source pressure that may alter the $\mathrm{m} / \mathrm{z}$ transmission profile, or differences in relative adduct formation propensities as a consequence of source maintenance, mobile phase composition, or glassware alkali metal content. Here we have taken into account this variability by defining a scaled average internal standard corrected response (2), calculated for each batch, $b$;

$$
X_{p, q c, b}^{\prime}=\frac{<X_{I S, q c, b}>}{<X_{p, q c, b}>} \frac{X_{p, q c, b}}{X_{I S, q c, b}}, \quad b=1,2, \ldots, 6
$$

$X_{p, q c}^{\prime}$, in equation (1) is then given by the vector (3).

$$
X_{p, q c}^{\prime}=\left(X_{p, q c, 1}^{\prime}, X_{p, q c, 2}^{\prime}, \ldots, X_{p, q c, 6}^{\prime}\right)
$$

Equation 1 does then not depend on the magnitude of batch to batch differences in average corrected response, and is equivalent to the RSD calculation defined by van der Kloet $e t$ $a l .{ }^{15}$ for a single batch study, or for a multi-batch study showing identical batch averaged corrected responses, as may be expected for example when using spectroscopic techniques. Optimal internal standards found for each variable using Equation 1 are listed in Supplementary Table 2A. 
Linear regression (internal standard corrected response as a function of sample injection order) was used to estimate for each variable in the QC calibration samples any intra-batch drift not corrected for by internal standard correction, as described by van der Kloet et al. ${ }^{15}$. For all variables, internal standard corrected response in each batch was divided by its corresponding intra-batch drift function, such that normalized variable abundance values in the study samples were expressed with respect to the batch averaged QC calibration serum samples (arbitrarily set to 1 ).

Following normalization, the concordance between duplicate sample injection response values was assessed. It was found that less than $0.1 \%$ of the repeat injections values where disparate $(\% \mathrm{CV}>30)$. In these cases, the corresponding sample injection data were returned for manual inspection of the automated integration performed by the TargetLynx software, and modifications performed where appropriate.

For identified metabolites, representative detector response curves were generated using a standard compound for each chemical class included in the analysis. By assuming similar detector response functions for all metabolites belonging to a given chemical class, a linear detection range could be defined for each variable. Minimum values were taken as those for which a signal-to-blank noise ratio of at least 5:1 was obtained, while maximum values were defined as those at which the detector response became nonlinear with respect to the concentration of the standard compound. Variable study data points lying outside their corresponding linear detection range were replaced with missing values and did not figure in subsequent statistical analyses. Supplementary Figure 1 displays response curves for the 18 representative standard compounds, showing on separate axes for each chemical class, the response distribution of all included study data points. Variables were not considered for further analysis where more than 5\% of data points were found outside their corresponding linear detection range (these data are not shown in Supplementary Figure 1). Supplementary Table 2A lists for each variable the percentage number of study data points that were replaced with missing values. As expected, variables that were excluded from further analysis at this stage (29/540) were the most abundant species detected by their respective platforms, e.g. ChoE(18:2) [blood concentration 2mM (www.hmdb.ca)].

The reproducibility of the analysis process was assessed using the $5 \mathrm{QC}$ validation serum extracts included in each batch. These gave a measure of the combined analytical variation over all process components including metabolite extraction, recovery, derivatization, LC/ MS injection, and data processing. Corresponding intra- and inter-batch (calculated over batch averaged QC validation serum extracts) \%CVs are listed for all variables included in the analysis in Supplementary Table 2A. Additionally, the cumulative distribution of \%CV is provided in Supplementary Table 3, being the median inter-batch \%CV values 5.0, 6.1, 11.9 for platforms 1,2 , and 3 respectively.

\subsection{Statistical analysis}

A number of recent studies have shown evidence for a strong correlation between BMI and the serum levels of several groups of metabolites included in the current analysis, including amino acids, ether phospholipids, NEFA, and glycerolipids ${ }^{19-21}$. In order to control the confounding BMI variable all patients were classified into three cohorts for subsequent analyses, as defined by the world health organization (WHO) ${ }^{22}$ : lean/pre-obese, $<30 \mathrm{~kg} / \mathrm{m}^{2}$; obese class I-II, $30-40 \mathrm{~kg} / \mathrm{m}^{2}$; obese class III, $>40 \mathrm{~kg} / \mathrm{m}^{2}$; hereafter referred to as lean, obese and morbidly obese patient cohorts respectively. Following this stratification, patients' BMI (global mean $43.6 \pm 11.5 \mathrm{~kg} / \mathrm{m}^{2}$ ) did not differ significantly between the histological groups in any of the three cohorts (lean, Kruskal-Wallis statistic $(\mathrm{K}-\mathrm{W})=3.02$, pvalue $=0.22$; obese, $\mathrm{K}-\mathrm{W}=3.36$, $\mathrm{p}$-value $=0.19$; morbidly obese, $\mathrm{K}-\mathrm{W}=4.37$, $\mathrm{p}$-value $=0.11$ ). 
All subjects were randomly divided into estimation ( $80 \%$ of all subjects, $n=374)$ and validation ( $20 \%$ of all subjects, $n=93$ ) groups such that in each group there was an equal proportional representation of individuals belonging to each BMI/histology cohort. To assess the relationship between metabolites (or other clinical variables) and NAFLD patient histological groups, two-tailed Wilcoxon rank sum tests were used for two-sample tests: steatosis/normal liver, NASH/normal liver, and NASH/steatosis. Kruskal-Wallis rank sum tests were used for three-way comparisons: normal liver/steatosis/NASH. The threshold for significance was $\mathrm{p}<0.05$ for all tests. Patient NASH/steatosis histology group pertinence probabilities were calculated by performing random forest ${ }^{23}$ analyses on all significant metabolite variables for each BMI patient cohort in the estimation group. Relative importance scores for all variables included in the random forest models are listed in Supplementary Table 2A. Receiver operating characteristic (ROC) curve analysis was used to assess the predictive potential of the random forest models. Overall diagnostic accuracy for a given two-class comparison was given by the area under the ROC curve (AUC) with its associated standard error. Sensitivity, specificity, positive predictive value, and negative predictive value were calculated for several different cutoff points: (1) at maximum average diagnostic accuracy (number of patients correctly classified/total number of patients), (2) that at which sensitivity of 0.95 (probability that NASH patients were correctly classified) was achieved, and (3) that at which specificity of 0.95 (probability that subjects that do not have NASH were correctly classified) was achieved. Model validation was performed (1) in the validation group, and (2) in the entire dataset (estimation + validation group). In the latter case cross-validation using the jackknife method was performed with 10 subgroups such that the possibility of an unusually positive or negative validation subset could be assessed. Both separate BMI group (only for patients classified within a given BMI group: lean/pre-obese, obese class I-II, or obese class III) and BMI-stratified (all patients obtained by feeding patients' serum metabolic profile dataset into its corresponding BMI group random forest model) ROC analyses were performed.

All calculations were performed using R v.2.12.1 (R Development Core Team, 2010) with random forest and receiver operating characteristic $\mathrm{R}$ (randomForest, ROCR) packages.

\section{Results}

Clinical, biochemical, and liver histology data obtained from the study participants are summarized in Table 1. Patients included in the study were within a similar age group $(43.8 \pm 11.8$ years), with a predominance of female subjects (76\%). Comparing within each BMI cohort, there were no significant differences between the histology groups in fasting serum total cholesterol (lean, $\mathrm{K}-\mathrm{W}=1.38$, $\mathrm{p}$-value $=0.50$; obese, $\mathrm{K}-\mathrm{W}=4.14$, $\mathrm{p}$-value $=0.13$; morbidly obese, $\mathrm{K}-\mathrm{W}=3.84$, $\mathrm{p}$-value $=0.15$ ). Fasting glucose concentrations were higher in obese NAFLD patients as compared to normal liver subjects, reaching statistical significance in the obese class I-II cohort (lean, $\mathrm{K}-\mathrm{W}=0.89$, p-value $=0.64$; obese, $\mathrm{K}$ $\mathrm{W}=9.75$, $\mathrm{p}$-value $=0.01$; morbidly obese, $\mathrm{K}-\mathrm{W}=5.79$, $\mathrm{p}$-value $=0.06$ ). Patients with NAFLD had increased TAG levels as compared to normal liver subjects, though these did not reach statistical significance in any of the three BMI cohorts (lean, $\mathrm{K}-\mathrm{W}=4.58$, $\mathrm{p}$-value $=0.10$; obese, $\mathrm{K}-\mathrm{W}=0.55$, $\mathrm{p}$-value $=0.76$; morbidly obese, $\mathrm{K}-\mathrm{W}=0.02$, $\mathrm{p}$-value $=0.99$ ). Transaminase levels were elevated in NAFLD patients as compared to normal liver subjects in all three BMI cohorts (aspartate aminotransferase: lean, $\mathrm{K}-\mathrm{W}=22.66$, p-value $<0.001$; obese, $\mathrm{K}$ $\mathrm{W}=5.44$, $\mathrm{p}$-value $=0.07$; morbidly obese, $\mathrm{K}-\mathrm{W}=35.0$, $\mathrm{p}$-value $<0.001$; alanine aminotransferase: lean, $\mathrm{K}-\mathrm{W}=28.65$, p-value $<0.001$; obese, $\mathrm{K}-\mathrm{W}=9.68$, $\mathrm{p}$-value $=0.01$; morbidly obese, $\mathrm{K}-\mathrm{W}=9.74$, p-value $=0.01$ ).

The relative ion abundance levels of 415 lipids ( 81 glycerolipids, 9 cholesterol esters, 42 sphingolipids, 231 glycerophospholipids, 27 NEFA, 13 bile acids, 8 oxidized fatty acids, 4 
acyl carnitines - see Supplementary Table 2A for individual metabolite identification details), 18 amino acids, and 78 unidentified species were included for statistical analysis in the estimation group ( $80 \%$ of all subjects). The entire profile, containing data points from each individual subject, is shown as a heat map in Supplementary Figure 2.

Figures $2 \mathrm{a}, \mathrm{b}$, and $\mathrm{c}$ depict average ion abundance ratios for the three different BMI patient cohorts, comparing steatosis/normal liver, NASH/normal liver, and NASH/steatosis groups respectively (metabolite class specific magnified representations of Figure 2 are provided in Supplementary Figure 3A-K, ion abundance ratios and statistical significance listed in Supplementary Table 2A). This figure indicates that serum metabolic changes reflecting NAFLD progression have a strong dependence on BMI. For instance, among morbidly obese patients most oxidized fatty acids are reduced in steatosis patients as compared to normal liver subjects, but are elevated in NASH relative to steatosis. The same species remain essentially unchanged when performing identical comparisons within the other BMI cohorts. NEFA show the reverse trend, again mostly restricted to the morbidly obese cohort, where levels of most species (including saturated, monounsaturated and polyunsaturated moieties - see Supplementary Figure 3I) are markedly increased in steatosis as compared to normal liver subjects, though significantly reduced in morbidly obese NASH with respect to steatosis patients. Several amino acids also show very similar reverted trends in the morbidly obese cohort: methionine is significantly increased, while serine, taurine, glutamic acid and aspartic acid are decreased in steatosis as compared to normal liver subjects; all five compounds show the opposite trend when comparing NASH to steatosis patients (Supplementary Figure 3J).

In addition to the above serum metabolite alterations found mainly in the morbidly obese group of NAFLD patients, we also observed considerable changes among some groups of metabolites that were exclusive to the lean patient cohort. Most sphingolipids were decreased in lean NASH patients as compared to individuals diagnosed with steatosis, with the most significant changes being observed amongst sphingomyelin species. No similar trend was found among the obese patient groups; indeed, several sphingolipids were significantly increased in morbidly obese NASH as compared to steatosis patients. Figure 3 details the groups of biomarkers which showed the most contrasting trends when comparing NASH to steatosis patients among the three BMI cohorts.

Besides the strongly obesity dependent changes already mentioned, a number of relative differences were found to be similar across all of the patients included in the study. As expected, all glycerolipid (including di- and triacylglyceride moieties) species were elevated in steatosis patients, as compared to normal liver subjects. We also observed that most of these species have reduced abundance levels in NASH as compared to steatosis patients, with the most significant changes being found among polyunsaturated fatty acyl (PUFA) containing species (Supplementary Figure 3A). Further groups of compounds that were found similarly altered in all three BMI patient cohorts include monoetherglycerophosphoethanolamine and monoetherglycerophosphocholine (including plasmalogen species - see Supplementary Figures 3E and 3G). These species were found decreased in steatosis patients as compared to normal liver subjects, with elevated levels in NASH. Finally, we also found that most bile acids included in the analysis were increased in NASH as compared to steatosis patients, with all species reaching statistical significance in the case of the morbidly obese individuals. In order to ascertain the predictive value of the serum metabolic profile for distinguishing between steatosis and NASH patients a random forest model was developed for each BMI cohort, using all $R t-\mathrm{m} / \mathrm{z}$ pairs significantly differentiating between the two groups. In total, 292 confirmed metabolites and 51 unidentified variables were significant, being 15, 9, and 237 of them specific to the lean, obese, and morbidly obese patient cohorts respectively. Significant variables are 
summarized in Supplementary Table 2B. ROC curves calculated for each model are shown in Figure 4(a), (b) and (c) for the lean, obese, and morbidly obese patient groups respectively. In the estimation group of patients the AUC was $0.82 \pm 0.04,0.83 \pm 0.03$, and $0.87 \pm 0.02$ for the lean, obese and morbidly obese groups of patients respectively. Defining the optimum cutoff point as that at which maximum average diagnostic accuracy (number of patients correctly classified/total number of patients) was obtained, estimation group sensitivity/specificity values were $0.67 / 0.89,0.66 / 0.95$ and $0.69 / 0.96$ for the lean (cutoff $=0.53$, accuracy $=0.82$ ), obese (cutoff $=0.51$, accuracy $=0.83$ ) and morbidly obese (cutoff $=0.57$, accuracy $=0.87$ ) cohorts respectively. Very similar AUC and sensitivity/ specificity values were obtained for the validation group and the full cross-validated dataset, as is also shown in Figure 4.

Next, the overall diagnostic accuracy of the BMI-dependent metabolic profile was assessed for all patients included in the study. This was achieved by feeding each test sample metabolic profile dataset into its corresponding BMI model and assessing the output with a global, BMI-stratified ROC analysis. Average model accuracy for classifying NAFLD patients (NASH/steatosis) is plotted as a function of cutoff value in Figure 5a; at maximum average accuracy the model predicted NASH with sensitivity/specificity $0.71 / 0.92$ in the estimation cohort (cutoff $=0.54$, accuracy $=0.82$ ), $0.56 / 0.89$ in the validation cohort (cutoff $=0.43$, accuracy $=0.77$ ), and $0.62 / 0.97$ in the full cross-validated dataset (cutoff $=0.57$, accuracy $=0.85$ ). Corresponding BMI-stratified AUCs were $0.87 \pm 0.02$ in the estimation group, $0.85 \pm 0.04$ in the validation group, and $0.84 \pm 0.01$ in the full cross-validated dataset. (Figure 5b). At maximum average accuracy the model was therefore able to correctly predict in the estimation group the absence of NASH in 186/198 patients, while of the 228 patients classified as steatosis 42 patients were incorrectly assigned. Hence the absence of NASH as predicted by the model was correct in $186 / 228$ cases (negative predictive value $=0.82$ ). The same cutoff criterion correctly predicted NASH in 62/104 patients, while of the 74 patients classified as NASH 12 were incorrectly assigned. The presence of NASH as predicted by the model was therefore correct on 62/74 occasions (positive predictive value $=0.84$ ). Two additional cut-off points were selected to achieve sensitivity of 0.95 (probability that NASH patients are correctly classified) or specificity of 0.95 (probability that subjects that do not have NASH are correctly classified) in the estimation group. Applying the low cutoff point $(<0.09), 71 / 198$ individuals without NASH were correctly identified, whereas $5 / 71$ subjects were incorrectly classified as NASH patients. Thus, using this cutoff point, the absence of NASH could be established in 71/76 of patients (negative predictive value $=0.93$ ). By applying the high cutoff point (>0.73), 67/104 of NASH patients were correctly identified, whereas $4 / 71$ were incorrectly classified. Using this cutoff point therefore, NASH could be established in $67 / 71$ of patients (positive predictive value $=0.94$ ).

\section{Discussion}

According to the WHO, in 2008 more than 500 million adults were obese (BMI > $30 \mathrm{~kg}$ / $\left.\mathrm{m}^{2}\right)^{22}$. WHO further projects that by 2015 this number will have increased to approximately 700 million $^{22}$. Obesity is a major risk factor for chronic conditions such as cardiovascular disease, diabetes and NAFLD ${ }^{22}$. The prevalence of NAFLD is currently estimated to be between $20 \%$ and $30 \%$ in Western adults, rising to $90 \%$ in the morbidly obese $e^{2,24}$. Fortunately, only a small fraction of NAFLD patients develop cirrhosis and hepatocellular carcinoma ${ }^{2,6,7}$, although rising obesity prevalence may result in a corresponding increase in these more severe conditions, representing a major health risk ${ }^{7}$.

The histological manifestation of early stage NAFLD is the accumulation of readily visible droplets of TAG fat in hepatocytes, produced as a consequence of increased hepatic NEFA influx that cannot be matched by $\beta$-oxidation or very low density lipoprotein (VLDL) 
secretion removal pathways ${ }^{2,3}$. An accumulating body of evidence suggests that TAG stored in this way is biologically inert and as such harmless. Indeed, hepatic TAG in NAFLD may have an adaptive, protective role in buffering toxic fatty acids that would otherwise be substrates for lipid peroxidation and oxidative stress, leading to hepatocyte damage, inflammation, cell death, and fibrogenesis ${ }^{4,5,25}$. This premise would seem to be supported by the fact that histological TAG may fade during NAFLD progression through NASH to cirrhosis $^{26}$. The current data show further evidence for this effect, indicating that circulating TAG levels are decreased in NASH as compared to steatosis patients. In all three BMI cohorts, the most significant of these changes were found among polyunsaturated fatty acid (PUFA) containing TAG.

Moreover, no significant differences were registered among any of the 12 TAG species containing exclusively saturated acyl chains. The contribution of TAGs derived from de novo lipogenesis is significantly increased in fatty liver individuals ${ }^{27,28}$. Decreased amounts of serum PUFA containing TAG observed in NASH in the current work may reflect accelerated hepatic de novo lipogenesis in these patients, leading to increased incorporation of saturated and monounsaturated fatty acids into VLDL particles, in turn producing decreased relative quantities of PUFA species in VLDL TAG ${ }^{29}$.

While triacylglyceride accumulation is now understood as a beneficial, adaptive response to the increased exposure of the liver to fatty acids, emerging evidence points to other fatty acid metabolites as being directly injurious to hepatocytes ${ }^{2,4,5}$. Oxidation represents a key mechanism for the removal of fatty acids through three different pathways: mitochondrial $\beta$ oxidation, peroxisomal $\beta$-oxidation, and endoplasmic reticulum (microsomal) cytochrome P450 enzymatic routes. Under normal circumstances most NEFA are metabolized through mitochondrial $\beta$-oxidation, though the extramitochondrial pathways become more important in conditions of NEFA overload or mitochondrial dysfunction, as is often associated with $\mathrm{NASH}^{30,31}$. Elevated serum levels of acyl carnitines are indicative of mitochondrial $\beta$ oxidation disorders ${ }^{32}$. Although the analytical platforms applied in the current work were not optimal for the coverage of the entire acyl carnitine profile, 3 out of 4 species observed as formate adducts in the methanol serum extract (platform 1) were significantly increased in morbidly obese NASH as compared to steatosis patients (Figure 3c). Peroxisomal $\beta$ oxidation may be adaptive when mitochondria are dysfunctional ${ }^{33}$; loss of the peroxisomal pathway in rodents causes NASH, while its stimulation has been found to be protective ${ }^{34-36}$. The current data show further evidence for altered peroxisomal activity in NASH patients, indicated by their significantly increased serum levels of monoetherphospholipids (including plasmalogens, synthesized in peroxisomes), as compared to individuals with steatosis. The clearest increases in these groups of compounds were also observed in the morbidly obese subject cohort where all but 2 of the 28 monoetherglycerophosphocholine and monoetherglycerophosphoethanolamine species profiled were significantly increased in NASH as compared to steatosis patients. Since plasmalogens can serve as antioxidants against reactive oxygen species ${ }^{37}$, elevated serum levels in NASH may also reflect increased activity of protective mechanisms against oxidative stress.

Further evidence for altered extramitochondrial oxidative activity, almost exclusively among morbidly obese patients, was found in the levels of oxidized fatty acids that were significantly higher in NASH as compared to steatosis patients for all 7 species identified using commercial standards. These included both enzymatic (15- and 12hydroxyeicosatetraenoic acid, pro-inflammatory lipoxygenase products) and nonenzymatic (5-, 9-, and 11-hydroxyeicosatetraenoic acid) oxidation products of arachidonic acid (Figure 3c). Perhaps partly as a consequence of the aforementioned elevated NEFA oxidative activity, most NEFA species were found significantly deregulated in morbidly obese NASH as compared to steatosis patients, except in the cases of arachidonic and eicosapentaenoic 
acid, where the reverse trends were found. A recent lipidomics study of human acquired obesity found increased adipose tissue levels of eicosapentaenoic acid, a precursor of antiinflammatory related docosahexaenoic acid (DHA) ${ }^{19}$. Elevated serum levels of eicosapentaenoic acid observed in the current work may similarly reflect its restricted conversion to anti-inflammatory DHA-derived lipid mediators in obese NASH.

Further changes mainly exclusive to the morbidly obese subject cohort were observed among essential amino acids, which were all significantly elevated in NASH as compared to steatosis patients except for tryptophan (not significant) and methionine that followed the reverse trend. Similar increases in serum essential amino acids have previously been related to a higher rate of whole-body protein turnover in NASH, modulated by co-factors such as cytokines, inflammation and insulin resistance ${ }^{38}$. While in the morbidly obese cohort of patients the clearest NASH metabolic abnormalities seem to be associated with the overloading of oxidative free fatty acid disposal routes, NASH is reflected in the lean cohort by significant alterations of a series of potentially lipotoxic intermediates that have been previously associated with NAFLD progression. The clearest evidence for this was found among the sphingolipid species where most ceramide and sphingomyelin species were found decreased in lean NASH as compared to steatosis patients (Figure 3a). Ceramides are thought to play a major role in lipotoxic cellular injury ${ }^{39}$, while sphingomyelin species have been previously associated with stress- and ligand-induced hepatocellular death, which contributes to the progression of several liver diseases including $\mathrm{NASH}^{40}$. Methionine is also found increased in lean NASH as compared to steatosis patients, mirroring the trend observed in lean rodent NAFLD models with disrupted one-carbon metabolism genes, methionine adenosyltransferase 1A (MAT1A-KO) and glycine N-methyltransferase knockout $(\mathrm{GNMT}-\mathrm{KO})^{41}$.

Besides providing platforms for the better understanding of disease pathogenesis, groups of serum metabolite biomarkers identified in the current study may be used to develop noninvasive tools for clinical NAFLD assessment. Evaluating this possibility, we built separate BMI-dependent multivariate models, showing that overall diagnostic accuracy (AUC) was greater than 0.8 in all three BMI cohorts. Feeding these data into an overall BMI-stratified model provided a maximum average diagnostic accuracy of 0.82 , at which point more than $90 \%$ of all NAFLD patients included in the study that did not have NASH could be correctly identified. The high accuracy of the model in ruling out the presence of NASH is particularly important, considering the fact that most NAFLD patients seen in clinical practice do not have advanced forms of the disease.

In summary we have studied the detailed serum metabolic profile of 467 liver-biopsied subjects, of which 377 were diagnosed with NAFLD. The measured NAFLD metabolic profile was dependent on BMI, an observation which indicates that the mechanism of NAFLD pathogenesis may be quite different depending on an individual's level of obesity. An imbalance between the supply to the liver of NEFA and their nontoxic disposal promotes lipotoxic injury that has been closely related to the pathogenesis of NAFLD progression ${ }^{2-5}$. This scenario may be reflected in the serum metabolome of obese NAFLD patients, where high serum NEFA abundances in steatosis patients are reverted in NASH in conjunction with elevated acyl carnitines indicating mitochondrial $\beta$-oxidation dysfunction, altered ether phospholipids reflecting disturbed peroxisomal function, and increased levels of proinflammatory eicosanoids providing further evidence of extramitochondrial oxidative activity. The alteration of enzymatic pathways that generate lipotoxic intermediates from NEFA or inhibition of pathways that dispose of them may also be speculated to promote lipotoxicity $2,4,5$. The lean NAFLD serum metabolome as characterized in the current work may carry the influence of this effect where species that have been previously linked to lipotoxicity, such as ceramides or sphingomyelin are found altered in NASH as compared to 
steatosis patients. The detailed mechanistic implications of the current data are likely to be complex and will require further study in future hypothesis-driven studies. In particular, it is unclear as to what extent metabolic changes occurring during NAFLD evolution are important in promoting progression of the disease or are manifestations of secondary phenomena. The use in the current work of state-of-the-art technology able to profile hundreds of individual metabolite species in parallel from large patient cohorts will help to provide the impetus for such work.

A drawback of the current approach is that we have not been able to provide absolute quantitation of the analytes, although we did monitor the reproducibility of our assays, while taking steps to ensure that all data points included in the analysis were within the linear detection range of the platforms. We would therefore expect the results to be qualitatively reproducible if a similar protocol were to be followed by an external laboratory, using the same sample set. The data show clear differential serum metabolic profiles associated with the groups of samples included in the study, offering strong possibilities for quantitative assay development needed for validation of the biomarkers. We also note the potential value of unidentified metabolites included in the qualitative profiling approach for providing valuable biomarkers that would otherwise remain unexplored.

Current means used for the diagnosis of NAFLD (i.e. liver biopsy or imaging techniques) are subject to significant uncertainty and are poorly suited as tests for such a prevalent condition, from both a clinical and financial point of view ${ }^{42,43}$. Imaging techniques are expensive and nonspecific, since they are unable to distinguish NASH from isolated steatosis, while liver biopsy is an invasive, subjective procedure, associated with potential complications and prone to sampling error ${ }^{44,45}$. Although most steatosis patients tend to have a benign, nonprogressive clinical course, a significant proportion of those with NASH show progressive liver disease with a significant associated risk of developing cirrhosis and its complications (portal hypertension, liver failure, and hepatocellular carcinoma) 2,6,7,24. Clearly then the distinction of NASH from steatosis is critical in order to identify high-risk patients and adapt their corresponding clinical management profile accordingly. Dyslipidemia, in particular hypertriglyceridemia has long been associated with the development of metabolic diseases such as NAFLD - the prevailing view being that excess bulk lipids are responsible for less favorable patient evolution. The present data, benefiting from the ability of modern technology to profile hundreds of diverse, intact lipid molecular species, indicate that a BMI-dependent serum metabolic profile distinguishes between NASH and steatosis patients, and may have significant implications for the development of biomarkers and potential novel targets for therapeutic intervention.

\section{Supplementary Material}

Refer to Web version on PubMed Central for supplementary material.

\section{Acknowledgments}

Financial Support

This work is supported by grants from PNI+D 2008-04800 and ETORTEK-2008 (J.M.M. and M.L.M.-C.), FIS PI10/00067 and Fundación Eugenio Rodríguez Pascual (C.G-M), NIH AT-1576 (S.C.L., M.L.M.-C. and J.M.M.), INTEK 06-20, ETORGAI-2008 IEI08-3, PS-010000-2008-5and FIT-06-101 (JB), FIS PI060085 (J.C.), HEPADIPEULSHM-CT-205 (J.M.M., M.L.M.-C., J.B., Y.L.M.B., P.G., K.C., J.T. and M.V.-C.); CIBERehd is funded by the Instituto de Salud Carlos III.

The contribution to this work from the laboratory technicians Ziortza Ispizua, Jessica Arribas, Mónica Martínez, and Begoña Etxezarreta is gratefully acknowledged. 


\section{List of abbreviations}

TAG

NEFA

NAFLD

NASH

UPLC-MS

BMI

TOF

MS/MS

CV

IS

RSD

WHO

K-W

ROC

AUC

VLDL

PUFA triacylglycerides

nonesterified fatty acids

nonalcoholic fatty liver disease

nonalcoholic steatohepatitis

ultra-performance liquid chromatography coupled to mass spectrometry

body-mass index

time-of-flight

tandem mass spectrometry

coefficient of variation

internal standard

relative standard deviation

world health organization

Kruskal-Wallis statistic

receiver operating characteristic

area under the ROC curve

very low density lipoprotein

polyunsaturated fatty acid

\section{References}

1. Frayn KN. Adipose tissue as a buffer for daily lipid flux. Diabetologia. 2002; 45(9):1201-1210. [PubMed: 12242452]

2. Angulo P. Nonalcoholic Fatty Liver Disease. N Engl J Med. 2002; 346(16):1221-1231. [PubMed: 11961152]

3. Cohen JC, Horton JD, Hobbs HH. Human Fatty Liver Disease: Old Questions and New Insights. Science. 2011; 332(6037):1519-1523. [PubMed: 21700865]

4. Bass NM. Lipidomic dissection of nonalcoholic steatohepatitis: moving beyond foie gras to fat traffic. Hepatology. 2010; 51(1):4-7. [PubMed: 20034031]

5. Neuschwander-Tetri BA. Hepatic lipotoxicity and the pathogenesis of nonalcoholic steatohepatitis: The central role of nontriglyceride fatty acid metabolites. Hepatology. 2010; 52(2):774-788. [PubMed: 20683968]

6. Matteoni CA, Younossi ZM, Gramlich T, Boparai N, Liu YC, McCullough AJ. Nonalcoholic fatty liver disease: a spectrum of clinical and pathological severity. Gastroenterology. 1999; 116(6): 1413-9. [PubMed: 10348825]

7. Starley BQ, Calcagno CJ, Harrison SA. Nonalcoholic fatty liver disease and hepatocellular carcinoma: A weighty connection. Hepatology. 2010; 51(5):1820-1832. [PubMed: 20432259]

8. Angulo P. Obesity and Nonalcoholic Fatty Liver Disease. Nutr Rev. 2007; 65:S57-S63. [PubMed: 17605315]

9. Machado MV, Cortez-Pinto H. No need for a large belly to have NASH. J Hepatol. 2011; 54(6): 1090-1093. [PubMed: 21281684]

10. Baker M. Metabolomics: from small molecules to big ideas. Nat Meth. 2011; 8(2):117-121.

11. Nicholson JK, Lindon JC. Systems biology: Metabonomics. Nature. 2008; 455(7216):1054-1056. [PubMed: 18948945] 
12. Kalhan SC, Guo L, Edmison J, Dasarathy S, McCullough AJ, Hanson RW, Milburn M. Plasma metabolomic profile in nonalcoholic fatty liver disease. Metabolism: clinical and experimental. 2010; 60(3):404-413. [PubMed: 20423748]

13. Puri P, Wiest MM, Cheung O, Mirshahi F, Sargeant C, Min H-K, Contos MJ, Sterling RK, Fuchs M, Zhou H, Watkins SM, Sanyal AJ. The plasma lipidomic signature of nonalcoholic steatohepatitis. Hepatology. 2009; 50(6):1827-1838. [PubMed: 19937697]

14. Barr J, Vazquez-Chantada M, Alonso C, Perez-Cormenzana M, Mayo R, Galan A, Caballeria J, Martin-Duce A, Tran A, Wagner C, Luka Z, Lu SC, Castro A, Le Marchand-Brustel Y, MartinezChantar ML, Veyrie N, Clement K, Tordjman J, Gual P, Mato JM. Liquid chromatography-mass spectrometry-based parallel metabolic profiling of human and mouse model serum reveals putative biomarkers associated with the progression of nonalcoholic fatty liver disease. J Proteome Res. 2010; 9(9):4501-12. [PubMed: 20684516]

15. van der Kloet FM, Bobeldijk I, Verheij ER, Jellema RH. Analytical error reduction using single point calibration for accurate and precise metabolomic phenotyping. J Proteome Res. 2009; 8(11): 5132-41. [PubMed: 19754161]

16. Kleiner DE, Brunt EM, Van Natta M, Behling C, Contos MJ, Cummings OW, Ferrell LD, Liu YC, Torbenson MS, Unalp-Arida A, Yeh M, McCullough AJ, Sanyal AJ. Design and validation of a histological scoring system for nonalcoholic fatty liver disease. Hepatology. 2005; 41(6):1313-21. [PubMed: 15915461]

17. Grumbach E, Wheat T, Mazzeo J. A novel method for the analysis of amino acids. Waters Application notes. 2007 Lib. Number WA43226.

18. Murphy RC, Fiedler J, Hevko J. Analysis of Nonvolatile Lipids by Mass Spectrometry. Chem Rev. 2001; 101(2):479-526. [PubMed: 11712255]

19. Pietiläinen KH, Róg T, Seppänen-Laakso T, Virtue S, Gopalacharyulu P, Tang J, RodriguezCuenca S, Maciejewski A, Naukkarinen J, Ruskeepää A-L, Niemelä PS, Yetukuri L, Tan CY, Velagapudi V, Castillo S, Nygren H, Hyötyläinen T, Rissanen A, Kaprio J, Yki-Järvinen H, Vattulainen I, Vidal-Puig A, Oreši3 M. Association of Lipidome Remodeling in the Adipocyte Membrane with Acquired Obesity in Humans. PLoS Biol. 2011; 9(6):e1000623. [PubMed: 21666801]

20. Fu S, Yang L, Li P, Hofmann O, Dicker L, Hide W, Lin X, Watkins SM, Ivanov AR, Hotamisligil GS. Aberrant lipid metabolism disrupts calcium homeostasis causing liver endoplasmic reticulum stress in obesity. Nature. 2011; 473(7348):528-531. [PubMed: 21532591]

21. Kim JY, Park JY, Kim OY, Ham BM, Kim H-J, Kwon DY, Jang Y, Lee JH. Metabolic Profiling of Plasma in Overweight/Obese and Lean Men using Ultra Performance Liquid Chromatography and Q-TOF Mass Spectrometry (UPLC-Q-TOF MS). J Proteome Res. 2010; 9(9):4368-4375. [PubMed: 20560578]

22. WHO . Obesity and overweight. 2011

23. Liaw A, Wiener M. Classification and Regression by randomForest. R News. 2002; 2(3):18-22.

24. Williams CH, Stengel J, Asike MI, Torres DM, Shaw J, Contreras M, Landt CL, Harrison SA. Prevalence of Nonalcoholic Fatty Liver Disease and Nonalcoholic Steatohepatitis Among a Largely Middle-Aged Population Utilizing Ultrasound and Liver Biopsy: A Prospective Study. Gastroenterology. 2011

25. Diehl AM. Genetic Susceptibility to Hepatic Steatosis. N Engl J Med. 2010; 362(12):1142-1143. [PubMed: 20335592]

26. Yeh MM, Brunt EM. Pathology of nonalcoholic fatty liver disease. Am J Clin Pathol. 2007; 128(5):837-47. [PubMed: 17951208]

27. Donnelly KL, Smith CI, Schwarzenberg SJ, Jessurun J, Boldt MD, Parks EJ. Sources of fatty acids stored in liver and secreted via lipoproteins in patients with nonalcoholic fatty liver disease. J Clin Invest. 2005; 115(5):1343-1351. [PubMed: 15864352]

28. Timlin MT, Parks EJ. Temporal pattern of de novo lipogenesis in the postprandial state in healthy men. Am J Clin Nutr. 2005; 81(1):35-42. [PubMed: 15640457]

29. Aarsland A, Wolfe RR. Hepatic secretion of VLDL fatty acids during stimulated lipogenesis in men. J Lipid Res. 1998; 39(6):1280-1286. [PubMed: 9643360] 
30. Pessayre D. Role of mitochondria in non-alcoholic fatty liver disease. J Gastroenterol Hepatol. 2007; 22:S20-S27. [PubMed: 17567459]

31. Sanyal AJ, Campbell-Sargent C, Mirshahi F, Rizzo WB, Contos MJ, Sterling RK, Luketic VA, Shiffman ML, Clore JN. Nonalcoholic steatohepatitis: Association of insulin resistance and mitochondrial abnormalities. Gastroenterology. 2001; 120(5):1183-1192. [PubMed: 11266382]

32. Bhuiyan AKMJ, Jackson S, Turnbull DM, Aynsley-Green A, Leonard JV, Bartlett K. The measurement of carnitine and acyl-carnitines: Application to the investigation of patients with suspected inherited disorders of mitochondrial fatty acid oxidation. Clin Chim Acta. 1992; 207(3): 185-204. [PubMed: 1327583]

33. Thyfault JP, Rector RS, Uptergrove GM, Borengasser SJ, Morris EM, Wei Y, Laye MJ, Burant CF, Qi NR, Ridenhour SE, Koch LG, Britton SL, Ibdah JA. Rats selectively bred for low aerobic capacity have reduced hepatic mitochondrial oxidative capacity and susceptibility to hepatic steatosis and injury. J Physiol. 2009; 587(8):1805-1816. [PubMed: 19237421]

34. Bass NM. Three for the price of one knockout - a mouse model of a congenital peroxisomal disorder, steatohepatitis, and Hepatocarcinogenesis. Hepatology. 1999; 29(2):606-608. [PubMed: 9918944]

35. Fan C-Y, Pan J, Usuda N, Yeldandi AV, Rao MS, Reddy JK. Steatohepatitis, Spontaneous Peroxisome Proliferation and Liver Tumors in Mice Lacking Peroxisomal Fatty Acyl-CoA Oxidase. J Biol Chem. 1998; 273(25):15639-15645. [PubMed: 9624157]

36. Green RM. NASH-hepatic metabolism and not simply the metabolic syndrome. Hepatology. 2003; 38(1):14-17. [PubMed: 12829980]

37. Morand OH, Zoeller RA, Raetz CR. Disappearance of plasmalogens from membranes of animal cells subjected to photosensitized oxidation. J Biol Chem. 1988; 263(23):11597-11606. [PubMed: 3403548]

38. Kalhan SC. Fatty Acids, Insulin Resistance, and Protein Metabolism. J Clin Endocrinol Metab. 2009; 94(8):2725-2727. [PubMed: 19657046]

39. Unger RH. Minireview: Weapons of Lean Body Mass Destruction: The Role of Ectopic Lipids in the Metabolic Syndrome. Endocrinology. 2003; 144(12):5159-5165. [PubMed: 12960011]

40. Mari M, Fernandez-Checa JC. Sphingolipid signalling and liver diseases. Liver Int. 2007; 27(4): 440-50. [PubMed: 17403183]

41. Mato JM, Martinez-Chantar ML, Lu SC. Methionine metabolism and liver disease. Annu Rev Nutr. 2008; 28:273-93. [PubMed: 18331185]

42. Bohte A, van Werven J, Bipat S, Stoker J. The diagnostic accuracy of US, CT, MRI and US, CT, MRI and 1H-MRS for the evaluation of hepatic steatosis compared with liver biopsy: a metaanalysis. Eur Radiol. 2011; 21(1):87-97. [PubMed: 20680289]

43. Rockey DC, Caldwell SH, Goodman ZD, Nelson RC, Smith AD. Liver biopsy. Hepatology. 2009; 49(3):1017-1044. [PubMed: 19243014]

44. Ratziu V, Charlotte F, Heurtier A, Gombert S, Giral P, Bruckert E, Grimaldi A, Capron F, Poynard T. Sampling Variability of Liver Biopsy in Nonalcoholic Fatty Liver Disease. Gastroenterology. 2005; 128(7):1898-1906. [PubMed: 15940625]

45. Mato JM, Lu SC. Where are we in the search for noninvasive nonalcoholic steatohepatitis biomarkers? Hepatology. 2011; 54(4):1115-1117. [PubMed: 21898494] 


\section{Synopsis}

An obstacle to the development of metabolome-based NAFLD predictors has been the lack of large cohort data from biopsy-proven patients matched for key metabolic features such as obesity. We examined the serum metabolome of 467 biopsied individuals with normal liver histology $(n=90)$ or diagnosed with NAFLD (steatosis, $n=246$; NASH, $\mathrm{n}=131$ ), using ultra-performance liquid chromatography coupled to mass spectrometry (UPLC-MS). The figure depicts obesity dependent biomarkers revealed by the study. 
a)
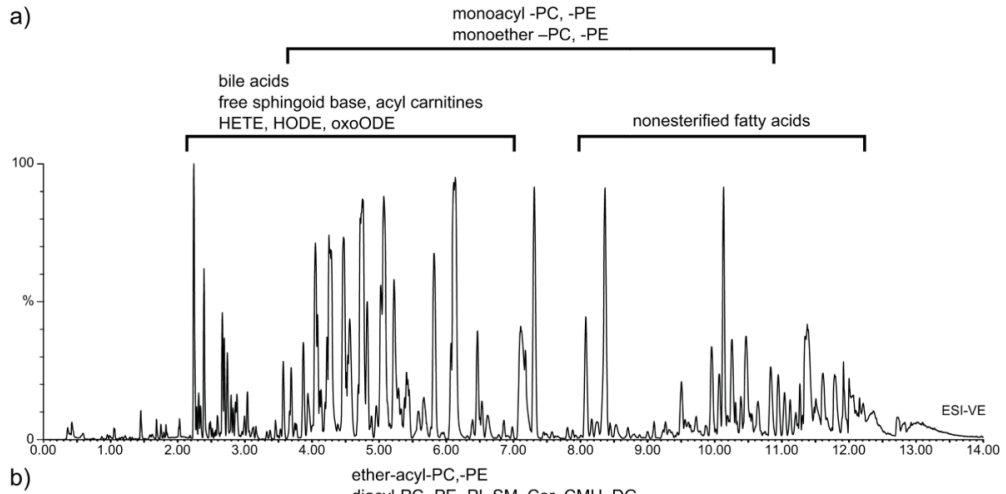

b) ether-acyl-PC,-PE
diacyl-PC,-PE,-PI, SM, Cer, CMH, DG

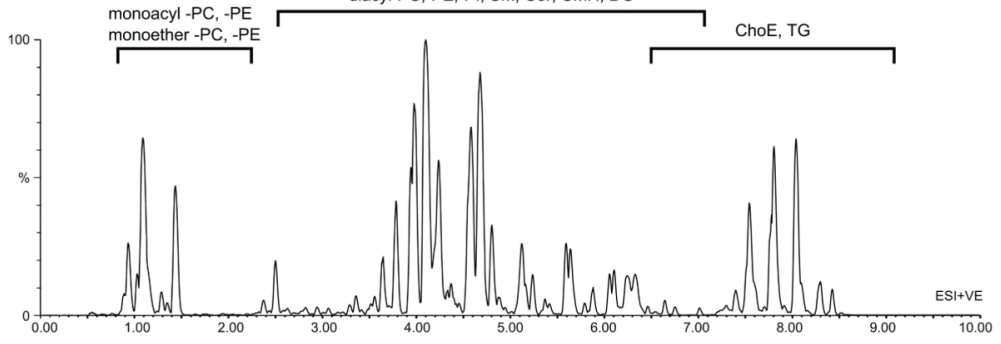

Figure 1. UPLC-TOF base peak ion intensity chromatograms

Base peak ion intensity chromatograms for the methanol - platform 1 (a), and chloroform/ methanol - platform 3 (b) serum extracts. Approximate retention time regions corresponding to identified metabolites are indicated on the plots (see text for abbreviations). 


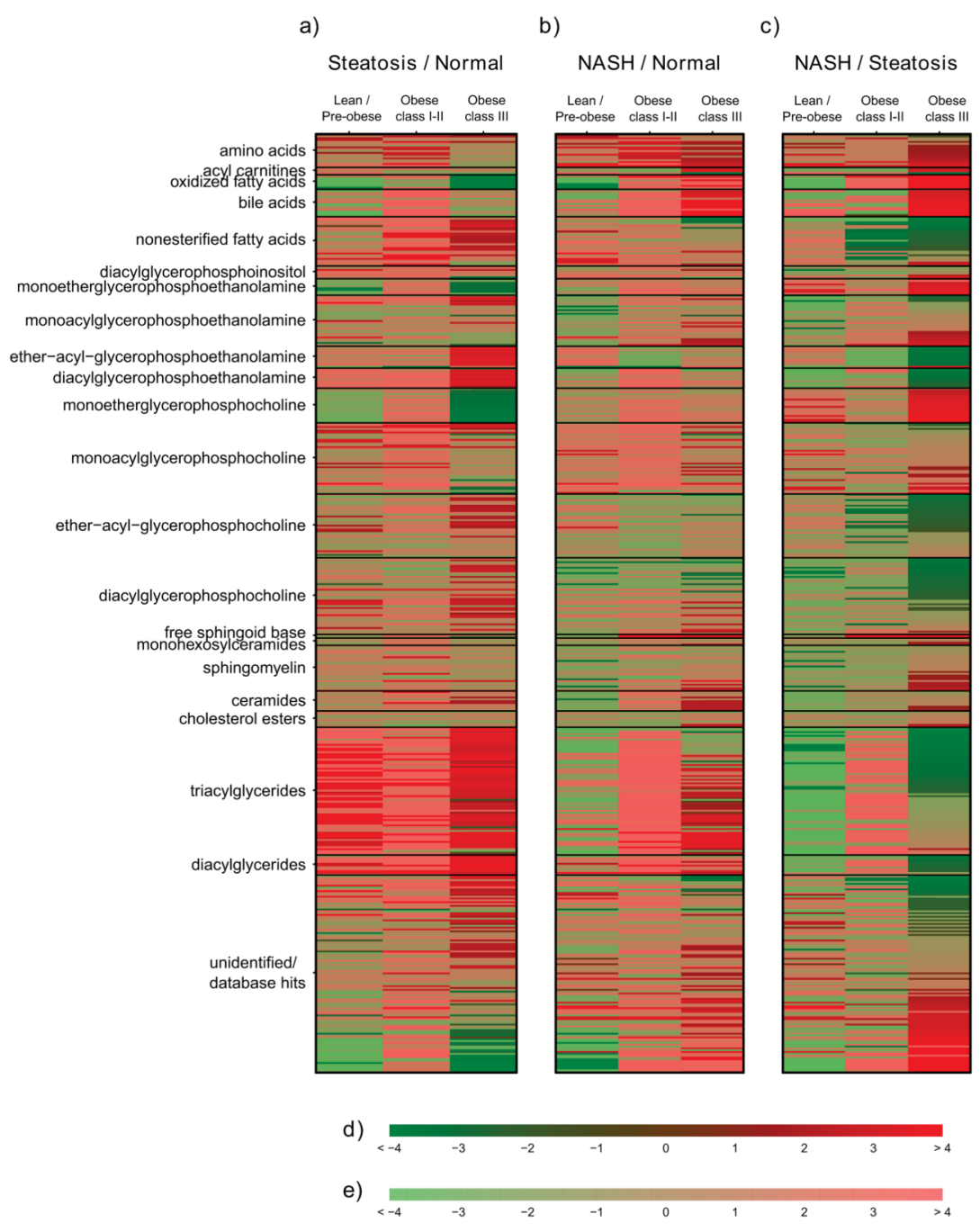

Figure 2. NAFLD serum metabolic profile

Heat map representation of the serum metabolic profile obtained from patients included in the study estimation group. (a), (b), and (c) metabolite ion abundance ratios in BMI cohorts lean/pre-obese (left), obese class I-II (middle), and obese class III (right), comparing histology groups: steatosis/normal liver, NASH/normal liver, and NASH/steatosis respectively. For each comparison, log transformed ion abundance ratios are depicted, as represented by the scales $(\mathbf{d})$, where pronounced colors correspond to significant $(\mathrm{p}<0.05-$ two-tailed Wilcoxon Rank Sum Test) changes, and (e) where light colors correspond to nonsignificant ( $p>0.05$ - two-tailed Wilcoxon Rank Sum Test) changes. Metabolite class specific magnified representations of Figure 2, showing individual metabolite details are provided in Supplementary Figures 3A-K. 
a)

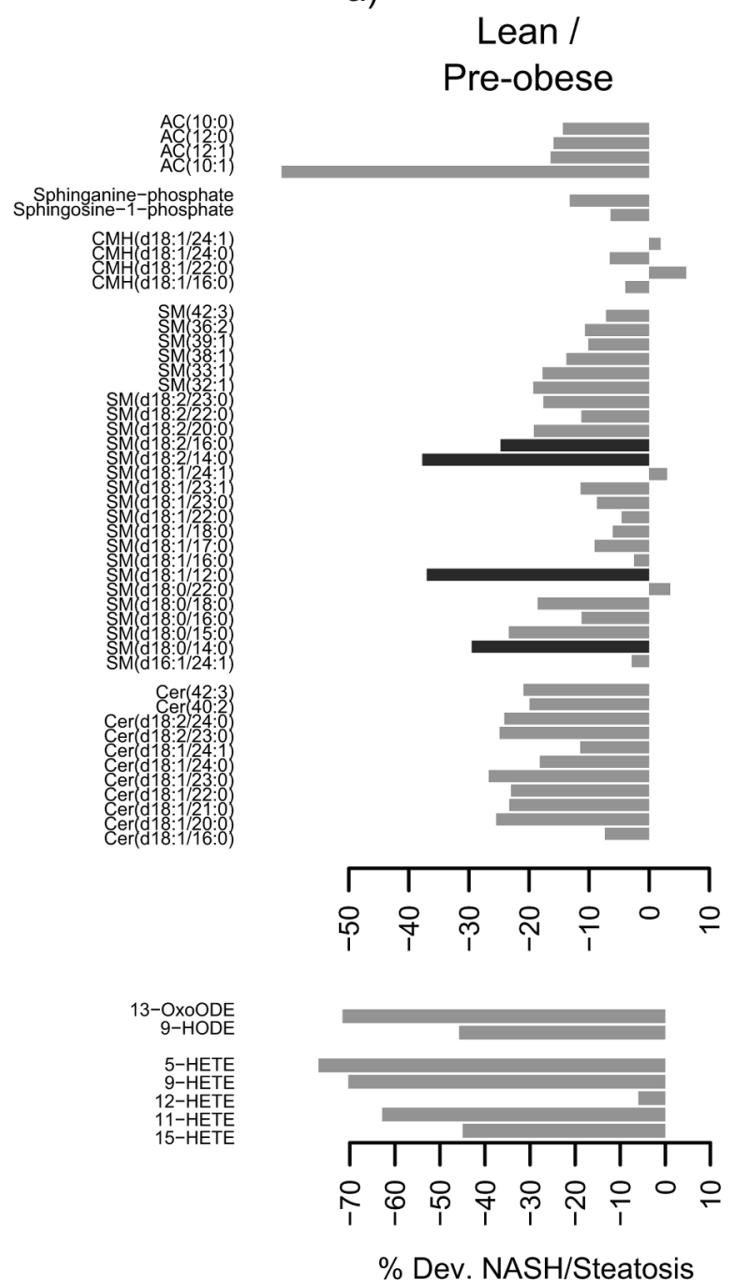

b)

\section{Obese \\ Class I-II}
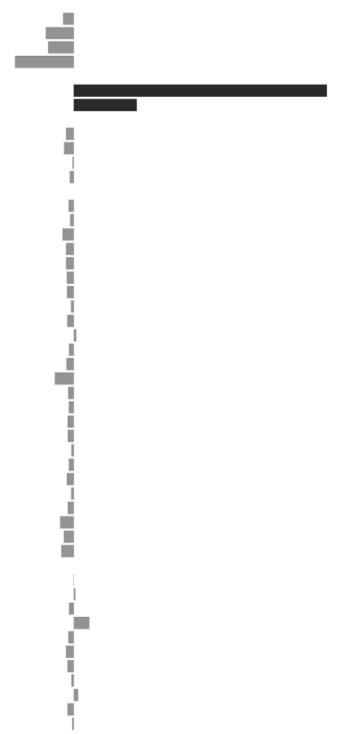

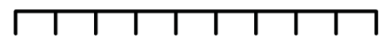

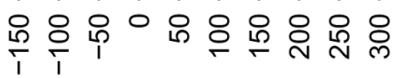

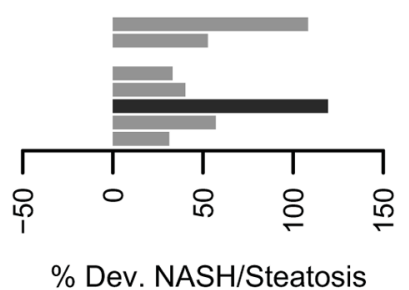

c)

\section{Obese \\ Class III}
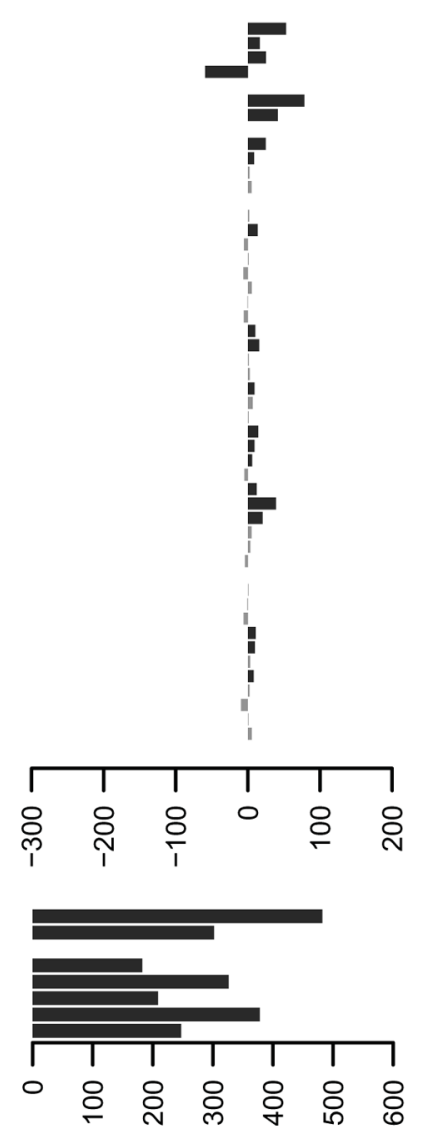

$\%$ Dev. NASH/Steatosis

Figure 3. Obesity dependent NASH biomarkers

Mean percent ion abundance deviations of acyl carnitines, sphingolipids (upper plots), and oxidized fatty acids (lower plots) found in the sera of patients diagnosed with NASH as compared to isolated steatosis. Data are shown for the lean/pre-obese (a), obese class I-II (b), and obese class III (c) patient cohorts. Positive and negative percentage values indicate higher levels of metabolites in NASH and steatosis patients' sera respectively. Dark bars denote significant changes ( $\mathrm{p}<0.05$, two-tailed Wilcoxon Rank Sum Test). 
a)

Lean / Pre-obese

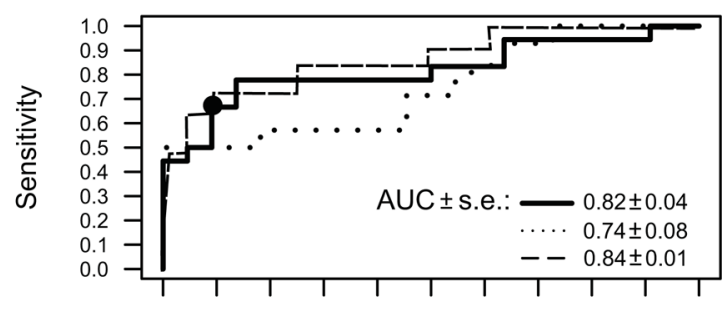

$\begin{array}{lllllllllll}0.0 & 0.1 & 0.2 & 0.3 & 0.4 & 0.5 & 0.6 & 0.7 & 0.8 & 0.9 & 1.0\end{array}$

1-Specificity

b)

Obese class I-II

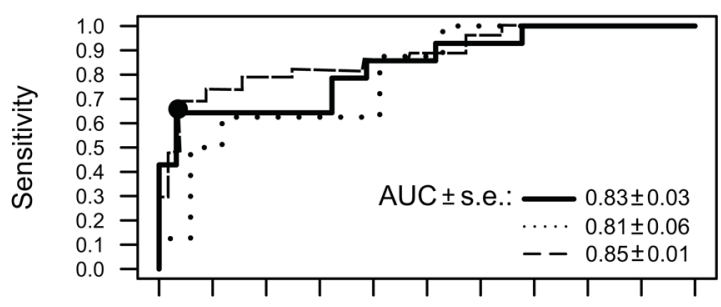

$\begin{array}{lllllllllll}0.0 & 0.1 & 0.2 & 0.3 & 0.4 & 0.5 & 0.6 & 0.7 & 0.8 & 0.9 & 1.0\end{array}$

1-Specificity

c)

Obese class III

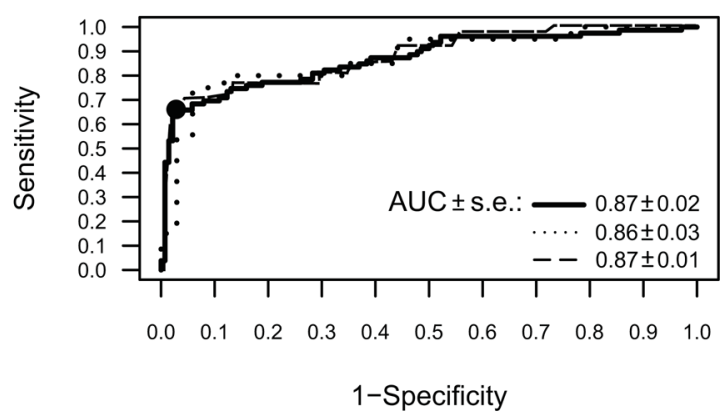

Figure 4. Obesity dependent metabolic discrimination between steatosis and NASH patients ROC curves calculated for the estimation (solid line), validation (dotted line), and full crossvalidated datasets (dashed line), based for each BMI cohort on all metabolite biomarkers found to be significant ( $\mathrm{p}<0.05$ - two-tailed Wilcoxon Rank Sum Test) in the estimation group. Data are shown for the lean/pre-obese (a), obese class I-II (b), and obese class III (c) patient cohorts. Optimum cutoff points (solid circles) are provided for each estimation group ROC curve. 
a)

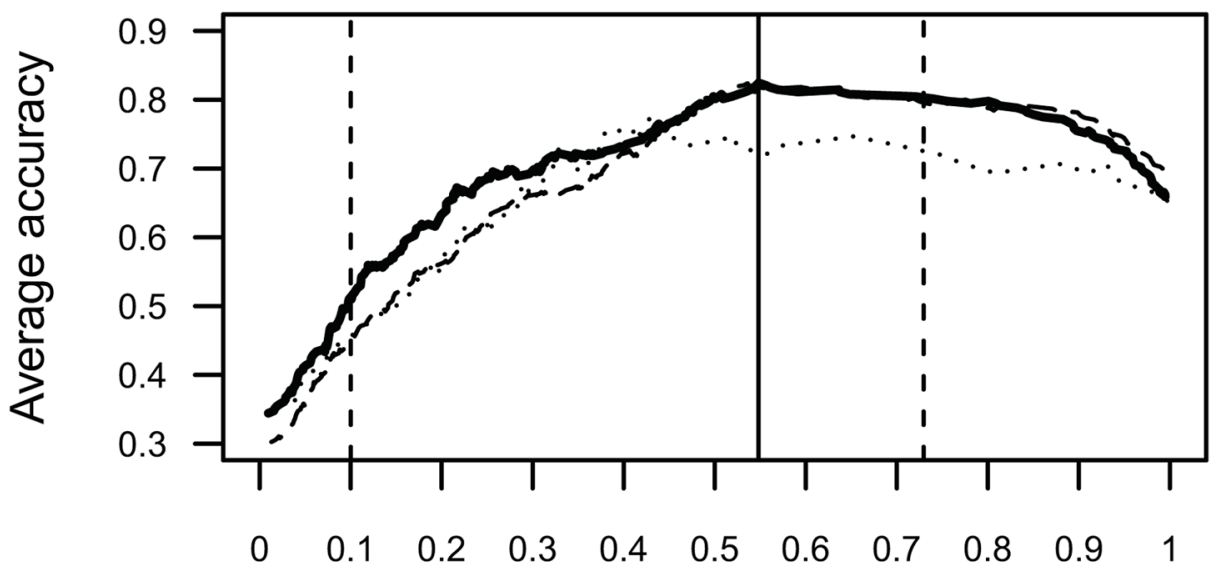

\section{Cutoff}

b)

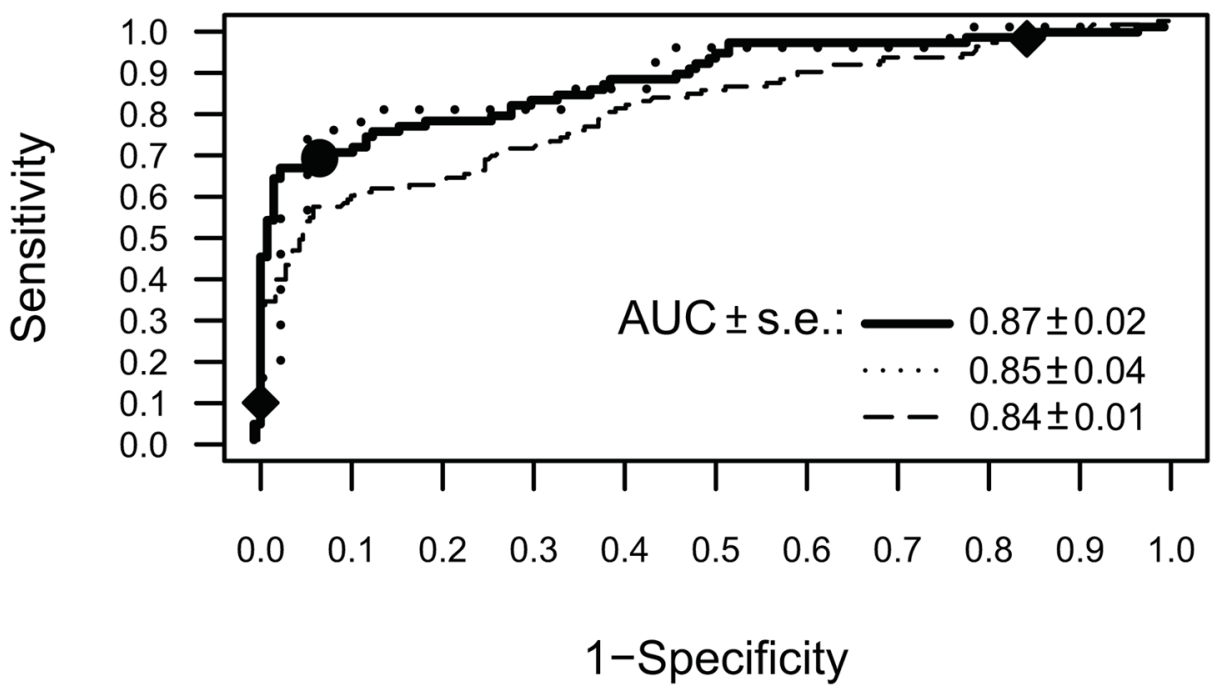

Figure 5. BMI-stratified metabolic discrimination between steatosis and NASH patients (a) Average BMI-stratified accuracy (number of patients correctly classified/total number of patients) as a function of cutoff for the three random forest models combined in the estimation (solid line), validation (dotted line), and full cross-validated datasets (dashed line). Estimation group cutoff points at maximum average accuracy (0.54), 95\% probability NASH absence (0.09), and 95\% probability NASH presence (0.73) are shown. (b) Associated BMI-stratified ROC curves for the estimation (solid line), validation (dotted line), and full cross-validated datasets (dashed line). The optimum cutoff point for the estimation group (0.54), defined as that at which average diagnostic accuracy was a maximum is indicated (sensitivity 0.71 , specificity 0.92 ) by a solid circle. In addition, the low cutoff point (0.09) to predict the absence of NASH with a probability of $95 \%$ and the 
high cutoff point (0.73) to predict the presence of NASH with a probability of $95 \%$ are shown (solid diamonds). 
